\title{
Towards Fault-Tolerant Strategy in Satellite Attitude Control Systems: A Review
}

\author{
Hicham HENNA ${ }^{1}$, Houari TOUBAKH ${ }^{2}$, Mohamed Redouane KAFI $^{3}$, and Moamar SAYED-MOUCHAWEH ${ }^{4}$ \\ 1,2,3 Laboratoire de génie électrique, University Kasdi Merbah, Ouargla, 30000, Algeria \\ henna.hicham@univ-ouargla.dz. \\ houari.toubakh@univ-ouargla.dz \\ kafi.redouane@univ-ouargla.dz \\ ${ }^{4}$ Mines-Douai, Douai, IA, F-59500, France \\ moamar.sayed-mouchaweh@imt-lille-douai.fr
}

\begin{abstract}
Spacecrafts are known to be very complex engineering systems where many technological devices enter in interaction to guarantee the overall mission objectives. Regardless of those sophisticated manufacturing systems, faults/failures are inevitable during the satellite lifetime. This paper aims at discussing the state-of-the-art approaches proposed to guarantee the satellites' attitude control system (ACS) performances when its components suffer from faults. The goal is to highlight their limits to address the specific challenges related to ACS. To localize and identify the potential faults, fault detection and diagnosis (FDD) methodologies are used in an earlier stage before the adaptation of the controller. The so-called fault-tolerant control (FTC) schemes have proven their capabilities during the last decade; nevertheless, those schemes still face some challenges that reduce their optimal performance in the aerospace industry. The contribution of this paper is to highlight the motivations and challenges of FTC/FDD methodologies when used to design spacecraft attitude controllers in the case of actuators and sensors anomalous behavior and to propose hints to address them.
\end{abstract}

\section{KEY WORDS:}

Fault detection and diagnosis (FDD), fault tolerant control (FTC), attitude control system (ACS), robust control, optimization.

\section{INTRODUCTION}

During the last two decades, space applications became more suitable for different types of military and civil services such

Hicham HENNA et al. This is an open-access article distributed under the terms of the Creative Commons Attribution 3.0 United States License, which permits unrestricted use, distribution, and reproduction in any medium, provided the original author and source are credited. as earth observation, meteorological forecasting, and spacebased science laboratories. Microsatellites represent an efficient tool to achieve the goals of the aforementioned missions due to their reduced cost in the design, manufacturing, and in-orbit exploitation phases. Microsatellites are commonly designed to operate at Low Earth Orbits (LEO), at which the exogenous disturbances such as atmospheric drag, gravity gradient, and earth magnetic field perturbation, have an important impact on attitude stabilization. Another risk that exists in this environment consists of space debris. Due to the above environmental factors combined with some physical systems readiness to fail especially the mechanical ones, faults/failures may occur during in-orbit exploitation of spacecraft. In the modern space industry and despite the improvement of components' quality, the number of emerging faults increases due to the increasing complexity of systems. The aforementioned faults consist of three categories: system faults, actuator faults, and sensor faults. Those faults are critical to the satellite attitude control for many reasons:

- Poor attitude determination quality in the case of sensor faults. Therefore, the guidance will diverge, which leads sometimes to trigger satellite safe mode to guarantee payload safety.

- Poor controllability when actuator faults occur; in which case, the attitude control loop performance (rise time, precision, .etc.) decreases accordingly.

- Another conceivable though rare scenario consists of software (process) faults such as division by zero or some parameters' anomalous normalization (e.g. quaternion).

We focus in this paper on the investigations about sensor and actuator faults. In Figure 1, several types of sensor faults are 
presented, while actuator faults are shown in Figure 2

(Ducard, 2009).

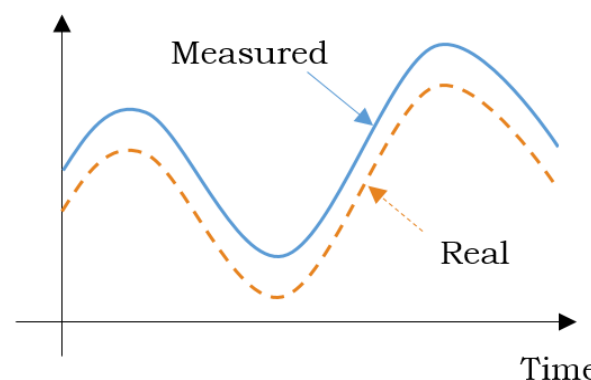

a)

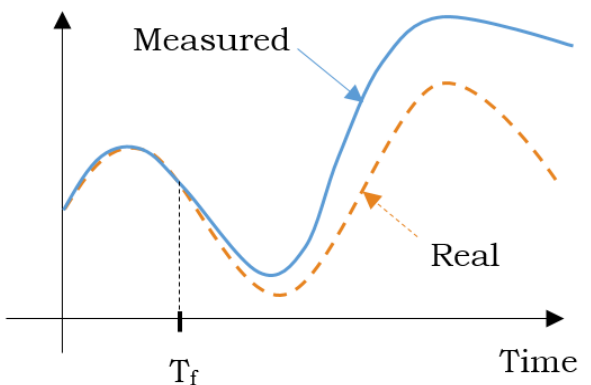

b)

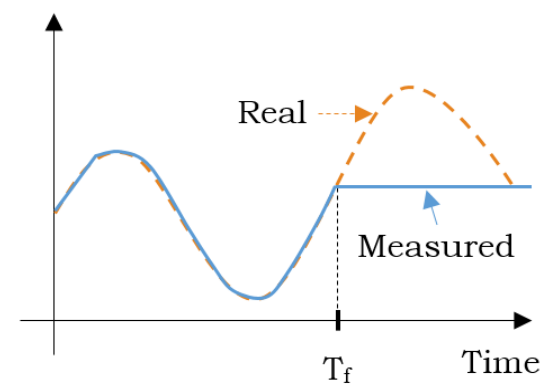

c)

Figure 1. Various types of sensor faults: (a) bias; (b) drift; (c) frozen sensor.

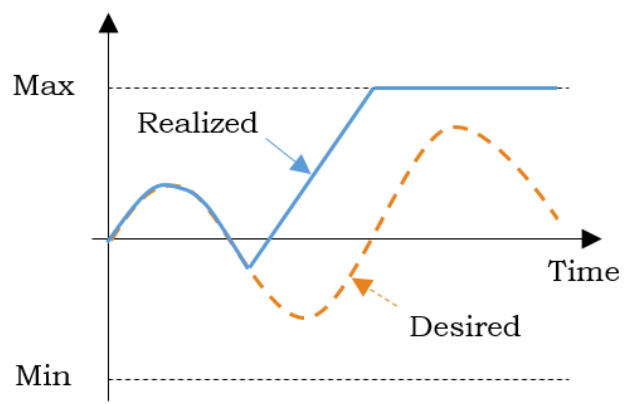

a)

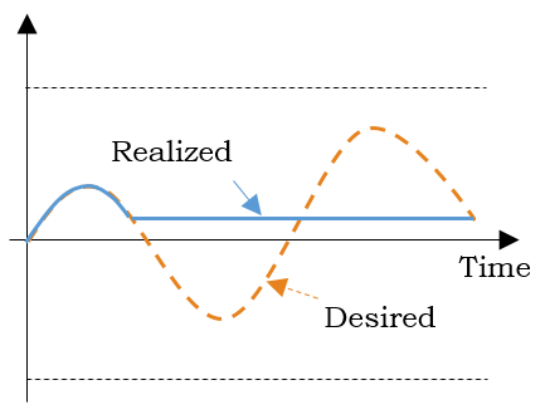

b)

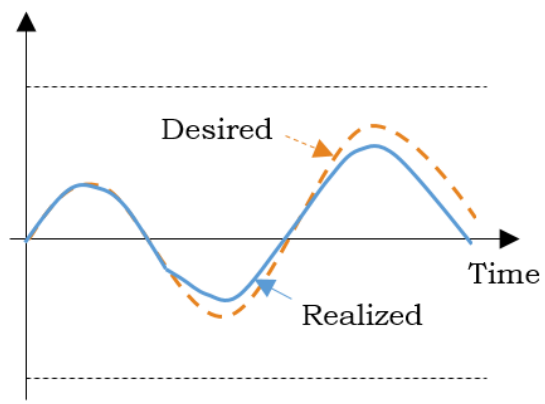

c)

Figure 2. Various types of actuator faults: (a) hard-over; (b) locked-in-place; (c) loss of effectiveness.

Before proceeding with the remainder of this introduction, it is convenient to give some real-life examples of component's faults to assimilate the severity of those faults towards space missions. The first mission consists of the SOlar and Heliospheric Observatory (SOHO). Series of gyroscope's calibration malfunctions led to full mission interruption between Jun. 25 and Oct. 24, 1998. After extensive recovery operations led by joint NASA/ESA collaboration, the spacecraft regain its normal sun pointing mode (mission recovery timeline is illustrated in Figure 3). With the recommendation of the joint-team, a gyro-less operation mode was implemented on Feb 1. 1999 (Bulletin 97 of European Space Agency, 1999). Even though, the near loss of SOHO was caused mainly by misconducted ground crew reaction (e.g. the 7th switch to emergency sun reacquisition (ESR) mode is triggered after switching the gyro-B off judged faulty by the ground-), the precise fault detection and diagnosis could interpose and improve ground crew judgment.

For the second space mission, the consequences were more catastrophic (complete mission loss) for the Japanese X-ray observatory Hitomi launched on 17 Feb. 2016, and suffered

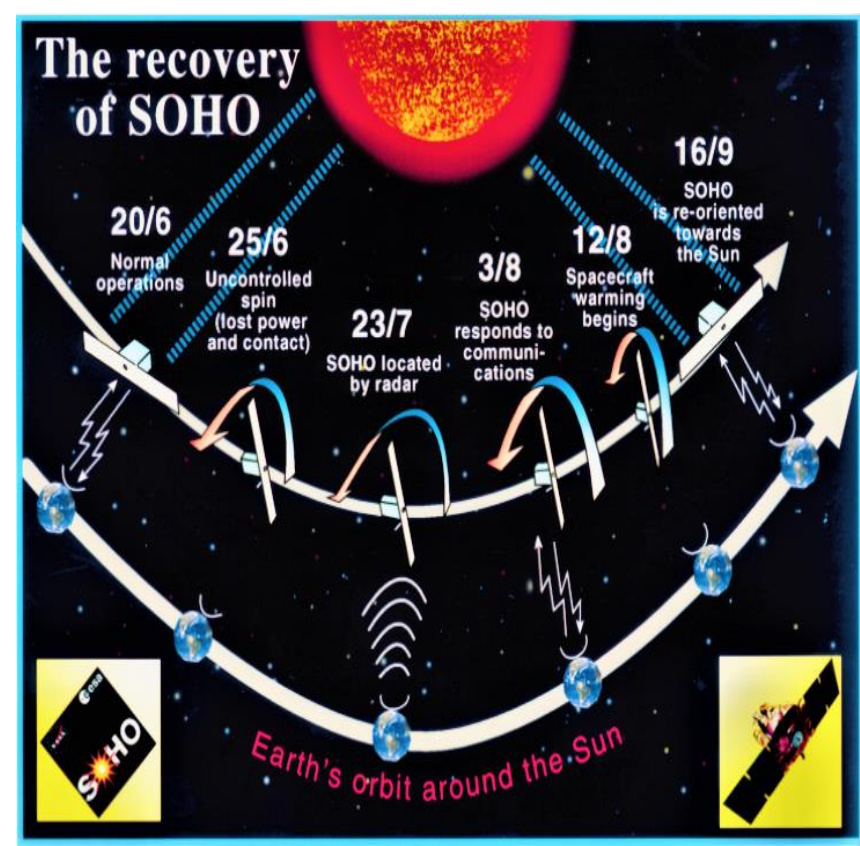

Figure 3. SOHO recovery timeline (ESA, 1999). 
from complete body break up into 10 pieces as reported by the U.S. Joint Space Operations Center (JSpOC) on 1 Apr. the same year. The investigations of the Japan Aerospace Exploration Agency (JAXA) confirm that attitude control loss on 25 Mar. caused by the Inertial Reference Unit (IRU) erroneous readings. Indeed, the inertial measurement unit (IMU) read $21.7 \% \mathrm{~h}$ rotation on $\mathrm{Z}$-axis while the spacecraft was actually stable (the Japan Aerospace Exploration Agency report, 2016). To counteract the non-existent spin, the ACS sent excessive control signals to be executed by the reaction wheels (RWs) at the early phase and the thrusters later. The excessive rotation rate led to a complete body break up on 26 Mar. only 39 days after launch!

As shown earlier, ACS elementary faults can lead to satellite mission unavailability due to safe mode transition as well as other catastrophic damages when control adaptation to a faulty situation is not carried out. Therefore, an alternative control concept that ensures mission availability side to side with acceptable performance in presence of faults has to be applied for satellite ACS, those control schemes are generally known as fault-tolerant control (FTC) systems. The basic idea behind FTC relies on achieving satisfactory control performance when system components suffer from faults/failures. The a priori design of FTC relies on attenuating the fault effect based on the controller robustness, we talk about passive FTC (PFTC). PFTC suffers from deep dependability of the FTC performance on pre-modeled faults, indeed, the performance decreases in the case of unanticipated faults. Moreover, hardware redundancy is sometimes needed to achieve FTC goals. To remedy the above drawbacks, another FTC strategy is elaborated, i.e., active FTC that firstly requires a fault detection and isolation (FDI) module to locate (which component is faulty) and identify (defining the fault characteristics such as severity, kind, etc.) a sensor or actuator fault. Sometimes the latter module is called fault detection and diagnosis (FDD). Then, based on the latter information, the controller is reconfigured to mitigate the fault effect on performance and stability (Alwi, Edwards, \& Tan, 2011), (Rouabah, Toubakh, \& Mouchaweh, 2020). A schematic of satellite active faulttolerant attitude control is drawn in Figure 4.

The application of fault-tolerant control (FTC) in satellite ACS design is very challenging due to the following factors (Zolghadri, Henry, Cieslak, Efimov, \& Goupil, 2014):

- Fast fault detection before degradation propagates throughout the system. The FDD performance depends also on fault criticality.

- Good fault isolation.

- Robustness to noises and uncertainties.

- High detection rate.

- Low false alarm rate.
- The basic FTC strategy relies on hardware redundancy which is limited due to weight and cost constraints. For example, in an agile microsatellite when three-axis stabilization is needed, the redundancy for angular velocities measurements requires to install three more gyroscopes!

- The feedback control robustness against noise and uncertainty could mask some components' faulty behavior which reduces fault detectability.

Moreover, FTC tasks (detection, reconfiguration) require to act in faulty situations without human supervision especially for LEO designed missions when ground station visibility slots are very limited (typically 45 minutes/day). On the other hand, for interplanetary or deep space missions, the time needed to establish the radiofrequency link (carrier of command and telemetry data) between the spacecraft and the ground station represents a major challenge for fast reaction in faulty situations. Furthermore, FTC strategies generally require more processing power when compared to the traditional PD or PID controllers which makes their conception w.r.t. satellite computing resources very challenging.

The outline of this paper is as follows. In the upcoming section, basics and classifications of FDD methodologies are provided. Then, an overview of model-based and data-driven FDD approaches is presented. Next, the major concepts and approaches of satellite attitude FTC are studied and compared according to aforementioned challenges. The conclusion is made in the last section by providing some guidelines on the design of an efficient and adapted FTC strategy for ACS.

\section{SPACECRAFT FAULT DETECTION AND DIAGNOSIS}

To accomplish the task of fault-tolerant control (FTC) efficiently, the first step of fault detection and diagnosis (FDD) has to be carried out. The FDD schemes for spacecraft attitude control system can be divided into three main features. First, the fault has to be detected using the fault detection mechanism. In the next stage, the faulty component is isolated after the detection of the fault. After that, the fault magnitude is obtained by fault identification. As shown in Figure 5, the basic idea behind FDD relies on residual generation followed by the decision (threshold-based) function. Based on the latter output, the reconfiguration mechanism is triggered.

Next, we review the FDD classification based on their synthesis principle and their research trends. A raw classification as reported in (Samy \& Gu, 2012) is illustrated in Figure 6. The availability of the spacecraft physical model is chosen as classification criteria. We further treat that aspect with additional criteria in the following paragraph. 
A classification of FDD methods as well as their referenced literature is shown in the Table 1.

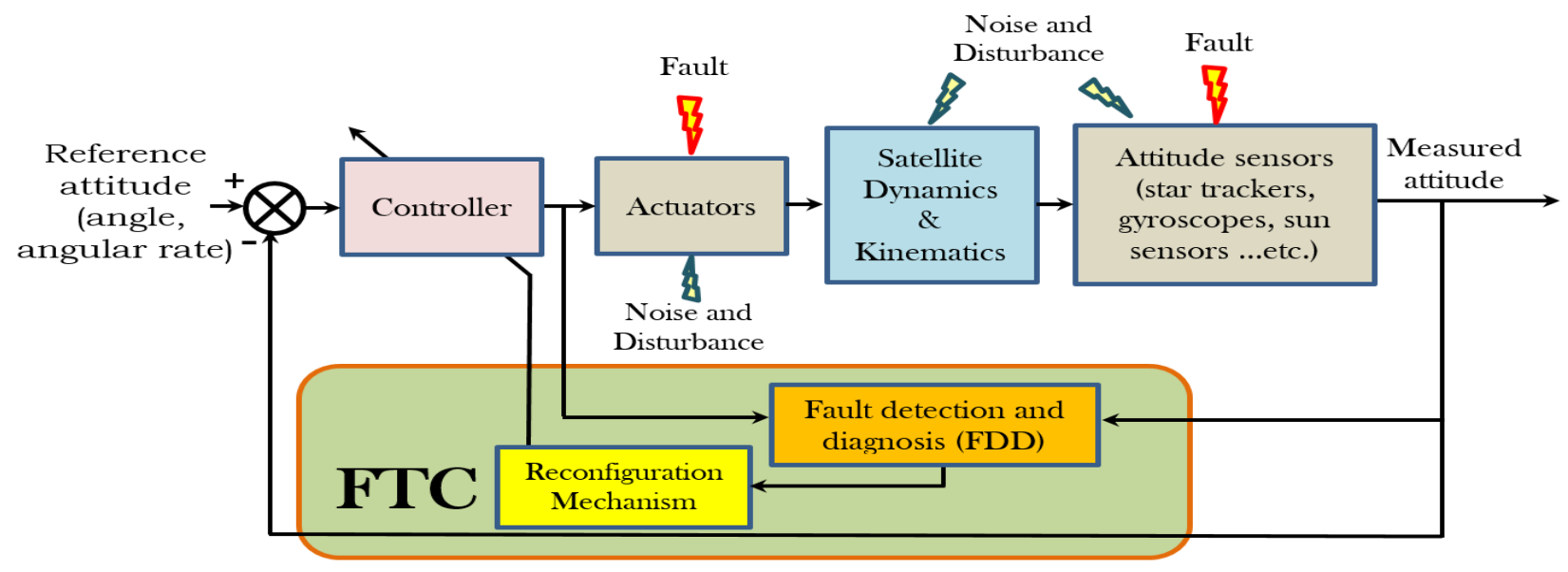

Figure 4. Active FTC scheme for satellite ACS.

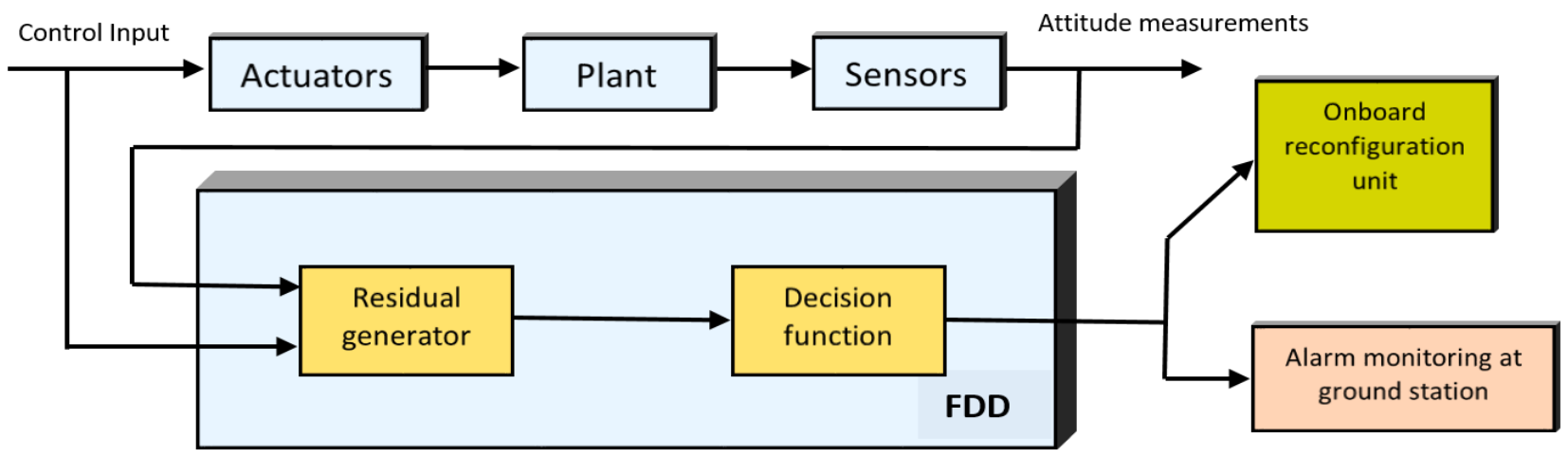

Figure 5. FDD principle (example: satellite attitude FTC).

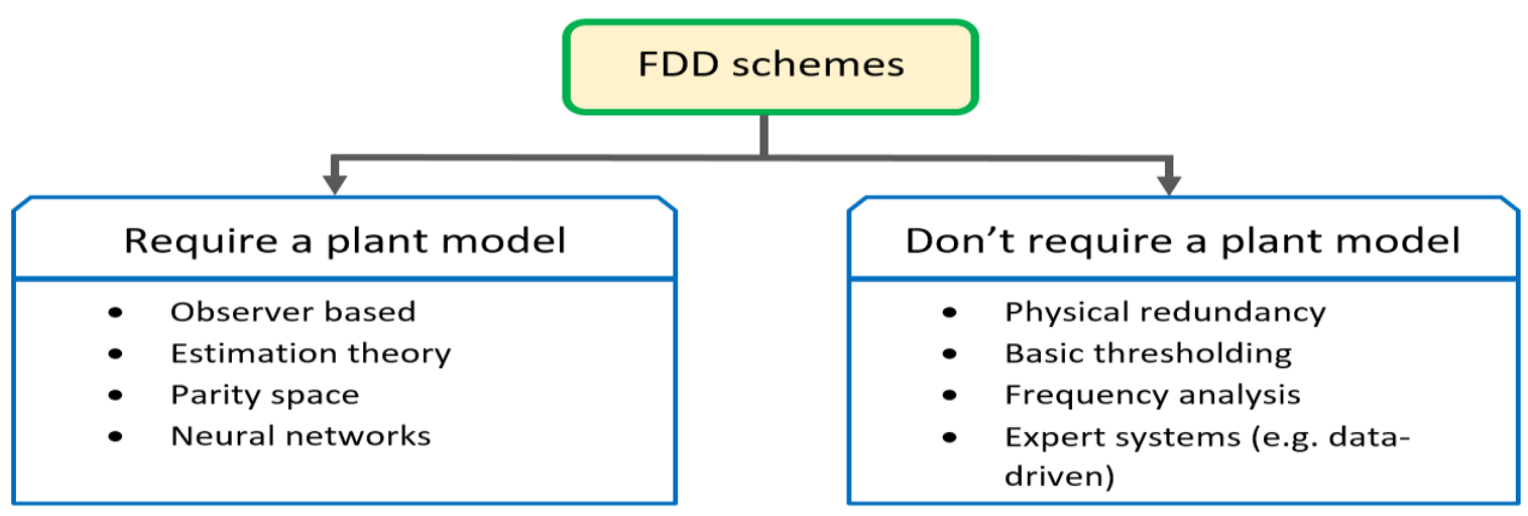

Figure 6. FDD classification (Samy \& Gu, 2012).

To give more insight into the aforementioned methodologies, we need to classify them based on functional comparison during design and exploitation phases, the following criteria are selected to establish that comparison:
- Physical knowledge: when the dynamic model is (poor ranking) or isn't (best ranking) needed. 
- Complexity: here we focus on a variety of space mission configurations in which a high complex spacecraft is built (deep space (e.g. Rosetta probe), space-lab...etc.).

- Autonomy: the ability to fulfill the requirements without human supervision.

- Computing consumption: the computing resources needed for FDD tasks execution.

- Non-stationarity: ability to separate abnormal behavior (caused by effective subsystems degradation) and environmental factors.

The classification based on the above criteria is presented in Table 2 . The ranking of each category is high (3 stars $* * *)$ when the corresponding technique satisfies with superiority the comparison criteria. For example, notice that poor autonomy ranking has been given to state estimation, that is, for KF-based estimation the filter gain is adjusted by ground crew in sensor-less reconfiguration.

In the upcoming subsections, an overview of recent developments in two main classes of FDD methods (namely model-based and data-driven) is outlined as well as the discussion of those methods in order to study their abilities and limitations.

\subsection{Model-based FDD}

Model-based FDD schemes dominate the FTC research field during the last two decades. In these schemes, the model of spacecraft dynamics is used to design the FDD algorithm. In (Djebko, Puppe, \& Kayal, 2019), a model-based FDD approach using the housekeeping telemetry is proposed. The nominal behavior is obtained using simulation. After that, the faulty behavior of satellite components is detected when a discrepancy between the expected and the observed behavior is raised. (Baldi, Blanke, Castaldi, Mimmo, \& Simani, 2018) proposes adaptive observers for actuator and sensor faults estimation. To avoid the effect of aerodynamic disturbance torques on fault estimation, a nonlinear geometric approach is adopted. Time-varying actuator faults were estimated using the sliding mode observer (SMO) proposed in (Gao Z. , Zhou, Qian, \& Lin, 2018). The asymptotical stability of the fault estimation observer was proven using Lyapunov theory. The SMO observers are insensitive to exogenous disturbances and parameter variations which justifies their wide use for spacecraft ACS fault-tolerant methods in aerospace research papers (Gao, Zhang, \& He, 2018), (Gao Z. , Zhou, Qian, \& Lin, 2018), (Alwi, Edwards, \& Marcos, 2010), (Nagesh \& Edwards, 2011). The proposed approach in (Gao S. , Zhang, Zhang, He, \& Lu, 2019) deals with satellite ACS sensor and actuator faults. Two extended Kalman filters (EKF) are used in attitude estimation. After that, using the attitude information delivered by EKFs, the system FDI is ensured. A gyroscope fault accommodation scheme was proposed in $(\mathrm{Li}$, Liu, Zhang, Wang, \& Shen, 2019). The studied case consists of a three axis gyroscopes installed orthogonally and one gyroscope installed with slantwise $(3 \mathrm{o}+1 \mathrm{~s})$. The measurement consistency is evaluated between the gyroscopes and the star sensor. This task is handled using a bank of dedicated Kalman filters. In (Lopez-Encarnacion, Fonod, \& Bergner, 2019), the problem of FDI for agile spacecraft was addressed.

Table 1. Popular techniques used to design FDD systems for ACS.

\begin{tabular}{|c|c|c|}
\hline & $\begin{array}{l}\text { FDD } \\
\text { Techniques }\end{array}$ & $\begin{array}{l}\text { Examples of papers } \\
\text { based on the technique }\end{array}$ \\
\hline \multirow[t]{3}{*}{$\begin{array}{l}\vec{D} \\
0 \\
0 \\
0 \\
\dot{0} \\
D_{0}^{0} \\
0 \\
0\end{array}$} & Sliding mode & $\begin{array}{l}\text { (Gao, Zhang, \& He, } \\
\text { Observer-Based Multiple } \\
\text { Faults Diagnosis Scheme } \\
\text { for Satellite Attitude } \\
\text { Control System, 2018), } \\
\text { (Gao Z. Zhou, Qian, \& } \\
\text { Lin, 2018), (Alwi, } \\
\text { Edwards, \& Marcos, } \\
\text { 2010), (Nagesh \& } \\
\text { Edwards, 2011) }\end{array}$ \\
\hline & $\#_{\infty}, \mathbb{Z}_{2}$ & $\begin{array}{l}\text { (Nemati, Safavi Hamami, } \\
\& \quad \text { Zemouche, 2019), } \\
\text { (Henry, 2008), }\end{array}$ \\
\hline & Adaptive & $\begin{array}{l}\text { (Baldi, Blanke, Castaldi, } \\
\text { Mimmo, \& Simani, 2018) }\end{array}$ \\
\hline \multicolumn{2}{|c|}{$\begin{array}{l}\text { Kalman filter/Extended } \\
\text { Kalman filter }\end{array}$} & $\begin{array}{l}\text { (Mehra, Rago, \& } \\
\text { Seereeram, 1998), (Gao S. } \\
\text {, Zhang, Zhang, He, \& Lu, } \\
\text { 2019), (Byeon, Mok, Woo, } \\
\text { \& Bang, 2019), (Li, Liu, } \\
\text { Zhang, Wang, \& Shen, } \\
\text { 2019), (Lopez- } \\
\text { Encarnacion, Fonod, \& } \\
\text { Bergner, 2019) }\end{array}$ \\
\hline \multicolumn{2}{|c|}{ Parity space } & $\begin{array}{l}\text { (Gao, Jiang, Shi, \& Cheng, } \\
\text { 2010) }\end{array}$ \\
\hline \multicolumn{2}{|c|}{ Fuzzy technique } & $\begin{array}{l}\text { (Wu S.-F., et al., 2001), } \\
\text { (Wu S.-F. , Engelen, } \\
\text { Babuška, Chu, \& Mulder, } \\
\text { 2003) }\end{array}$ \\
\hline \multirow{4}{*}{ 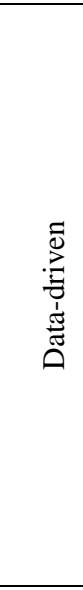 } & PCA & $\begin{array}{l}\text { (Ke-Qiang, et al., 2019), } \\
\text { (Li, et al., 2019) }\end{array}$ \\
\hline & SVM & $\begin{array}{l}\text { (Ke-Qiang, et al., 2019), } \\
\text { (Ibrahim, Ahmed, Zeidan, } \\
\text { \& Ziedan, 2019), (Hasan } \\
\text { Abbasi, Castaldi, } \\
\text { HamedDehghan, } \\
\text { Simani, 2019) }\end{array}$ \\
\hline & $\begin{array}{l}\text { Pattern } \\
\text { recognition }\end{array}$ & (Ke-Qiang, et al., 2019) \\
\hline & $\begin{array}{l}\text { Neural } \\
\text { Networks }\end{array}$ & $\begin{array}{l}\text { (Liu, Pan, Wang, \& He, } \\
\text { 2019), (Sun, Wang, He, } \\
\text { Zhou, \& Gu, 2019), } \\
\text { (Omran \& Murtada, 2017), } \\
\text { (Lee, Lim, Cho, \& Kim, } \\
\text { 2020) }\end{array}$ \\
\hline
\end{tabular}


Table 2. Comparison of FDD methods (used for satellite ACS).

\begin{tabular}{|l|c|c|c|c|c|}
\hline Techniques & Knowledge & Complexity & Autonomy & Computing res. & Non-stationarity \\
\hline Observer based & $*$ & $* *$ & $*$ & $* *$ & $* *$ \\
\hline State estimation & $*$ & $*$ & $*$ & $* *$ & $*$ \\
\hline Parity space & $*$ & $*$ & $*$ & $* * *$ & $*$ \\
\hline Fuzzy technique & $* *$ & $* *$ & $* *$ & $* *$ & $* *$ \\
\hline Data-driven & $* * *$ & $* * *$ & $* * *$ & $* *$ & $* * *$ \\
\hline
\end{tabular}

The proposed FDI scheme aims at detecting and isolating faults in thrusters, reaction wheels (RW), and RW tachometers. The fault detection is ensured with a generalized likelihood ratio (GLR) technique applied to the residual signal. For the FDI of a satellite formation flight, a nonlinear observer that minimizes the uncertainty within $\mathbb{\#}_{\infty}$ framework was proposed in (Nemati, Safavi Hamami, \& Zemouche, 2019). In the proposed scheme, each satellite in the constellation can diagnose its neighbor's faults. To predict the remaining useful life (RUL) of RWs motor in satellites, (Yun, Lee, Oh, \& Choi, 2019) proposes an EKF-based scheme. The proposed approach is divided into two EKF algorithms; those algorithms are used to predict the RW motor RUL. Another quantitative classification of model-based FDD approaches suggested by (Zhang \& Jiang, 2008) treats among others the aspect of suitability for FTC design as well as computational complexity. (Zhang \& Jiang, 2008) proposes that simultaneous/multiple parameter estimation (multiobservers, EKF, 2-stage KF) is more suitable for FTC applications. Nevertheless, they may be less suitable when computational power is limited.

\subsection{Data-driven FDD}

At the design level of space missions, a high fidelity spacecraft dynamic model may not be available. Model unavailability increases with the complexity of satellite flexible modes. That is, the use of model-based techniques to design the FDD scheme may not fulfill the satellite ACS performance requirements. To overcome that drawback, new FDD approaches need to be investigated. Data-driven methodologies represent one main alternative to model-based ones in that field, and for which many papers were shown in the literature during the last few years. For that aspect, it is shown previously in this paper (Table 2) that novel artificial intelligence approaches increase the computational burden w.r.t. parameter/state estimation schemes, which represents a major drawback of AI approaches implementation in microsatellite's software. From another point of view, modelbased approaches suffer from dependability on physical knowledge of both the dynamic model as well as the anticipated fault model. The aforementioned concepts continue to feed the debate about how to improve those strategies, either for model-based schemes to cope with many fault scenarios, or to alleviate the computational burden in the case of AI schemes with faster and more optimal algorithms.

Arthur Samuel defines (Hassanien, Darwish, \& El-Askary, 2020): "Machine learning (ML) is a tool of system science field that can learn from gathered sample input data and extract structural information for building a model". The previous definition means that for satellite's FDD, the telemetry (TM) data is used to construct datasets on which ML algorithms are applied. Meanwhile, ML space applications have some challenges linked to the space-ground data transfer: the amount of designed TM to be transmitted by the spacecraft is limited (sensor's installation for every conceivable parameter contradicts cost/space optimization paradigm). Furthermore, even for chosen TM, the number of data-samples per unit time is limited due to the communication bitrate boundaries. The categorization of ML as shown in Figure 7, can be divided into:

- The learning strategy used to obtain models,

- The task chosen to get the desired output.

In (Li, et al., 2019), kernel principal component analysis (KPCA) was used on a set of training data to analyze the correlation between some reaction wheels parameters. The proposed scheme can detect faults based on the abnormal changes in testing data and without any prior knowledge of the fault model. In (Ibrahim, Ahmed, Zeidan, \& Ziedan, 2019), Egyptsat-1 telemetry data was analyzed using Support Vector Machine (SVM) to extract components' nominal behavior patterns. Then, a fault diagnosis scheme based on Fault Tree Analysis (FTA) is performed to determine the most probable cause of the last 2010 satellite failure. Data feature extraction for FDD based on Supervised Local Linear Embedding (SLLE) was established in (Qu, et al., 2019). The proposed approach was performed on real satellite (Luojia1-01) telemetry data, to effectively enhance FDD capabilities. A novel model-free framework for RWs fault detection and isolation (FDI) was proposed in (Hasan Abbasi, Castaldi, HamedDehghan, \& Simani, 2019). The gyroscopic effects, measurement noise, and aerodynamic disturbances were considered. 


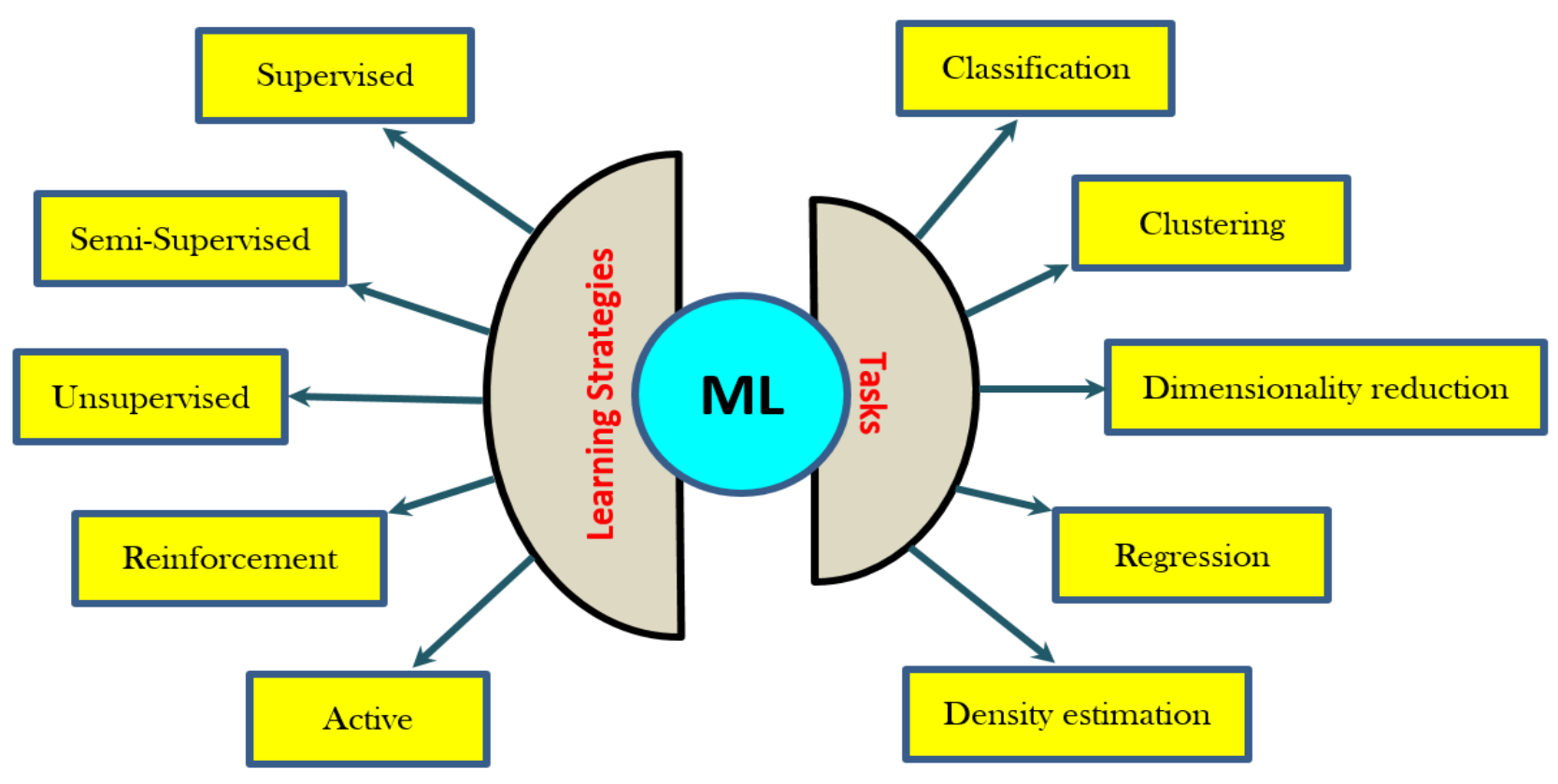

Figure 7. Machine learning categorization.

The introduced mixed learning scheme uses Random Forest (RF), Partial Least Square (PLS), SVM and Naïve Bayes to perform FDI tasks. In early phases of spacecraft components' reliability check, a long-life test is commonly performed on critical hardware such as moment wheel assemblies (MWAs). An MWA health monitoring data-driven approach was reported in (Wu, Wang, Liu, He, \& Xie, 2019) that relies on acoustic parameters. The so-called clustering fusion methodology aims at the feature vectors containing: root mean square (RMS), kurtosis, and sharpness parameters.

In addition to the preceding data-driven approaches, neural network (NN) schemes represent a powerful tool to carry out FDI plans. (Liu, Pan, Wang, \& He, 2019) proposes a RWs bearing FDI approach using a convolutional neural network $(\mathrm{CNN})$. The feature input maps rely on the time-domain signal of the rotating object vibration. Then, image recognition characteristics of $\mathrm{CNN}$ are called to handle bearing fault diagnosis. In (Sun, Wang, He, Zhou, \& Gu, 2019), the problem of the quick disappearance of faults' effect due to the closed-loop control system was investigated. The proposed FDI approach relies on deep neural networks (DNN). A DNN based on the sliding window technique is used to effectively differentiate the fault characteristics of several data modes. In (Lee, Lim, Cho, \& Kim, 2020), the problem of Nanosatellites' autonomous FDI was addressed. The proposed scheme uses deep learning techniques to detect and identify reaction wheels faults. A long-short term memory (LSTM) model was elaborated using the training data. The residuals are fed to the LSTM model to deliver the fault label to the satellite CPU.
It's shown from the previous papers that most of the validation results are obtained based on simulation. Furthermore, the faults and disturbances are considered to be theoretically bounded. Some simulation models are also simplified to a limited set of scenarios such as small attitude rotation angles (Gao Z., Zhou, Qian, \& Lin, 2018). One major obstacle for most spacecraft FDD developments (mainly for the academic community) is that there is no access to high fidelity simulators used by the dominating space organizations (NASA, ESA, CNES...etc.). According to (Wander \& Förstner, 2012), hierarchical fault detection, isolation, and recovery (FDIR) remains crucial to ensure spacecraft health management especially during critical phases (e.g. orbit insertion for deep-space probe). The FDD approaches studied in this paper can be applied at the $1^{\text {st }}$ and $2^{\text {nd }}$ levels of the hierarchical FDIR mainly for component's FDD. The remainder of hierarchical FDIR addresses the problems of high critical faults particularly when data transmission is very slow (spacecraft-earth large distances), and relies on some sophisticated techniques classified as industrial and do not fit the subject of this paper. For the architecture of spacecraft's hierarchical FDIR, please refer to Figure 2 in (Wander \& Förstner, 2012) and Fig. 7.1 in (Zolghadri, Henry, Cieslak, Efimov, \& Goupil, 2014).

Machine learning is relatively a recent discipline for control problems. Therefore, a comparative study is needed to highlight ML individual techniques that can be selected to address FDD/FTC problems for spacecraft ACS. Each ML method has advantages and disadvantages. Therefore, to increase the ML approach reliability, one can implement 
different scheme fusion as reported in (Hasan Abbasi, Castaldi, HamedDehghan, \& Simani, 2019).

In the following section, we will discuss the performance criteria of some popular ML approaches studied in academic literature during the last few years. For learning concept comparison (see Table 3), the chosen criteria consist of: Labelling and human supervision which can be seen as a single element because to get labeled features, the domain's expert feedback is needed.

Computation burden and non-stationarity are constant criteria throughout this paper.

The comparison between FDD techniques as stated in the following tables is based on recent studies about ML applications in addition to the author's analysis (Toubakh, 2015). Regarding the novelty of ML application in aerospace technologies, several studies discuss technological themes such as: remaining useful life (RUL) of batteries (Saha, Goebel, \& Christophersen, 2009), machine learning regression techniques for LiDAR-derived estimation (García-Gutiérrez, Martínez-Álvarez, Troncoso, \& Riquelme, 2015), and performance comparison of several ML techniques such as SVM and RF for intrusion detection (Ahmad, Basheri, Iqbal, \& Raheem, 2018). Some aforementioned papers treat as stated previously technological domains that are not explicitly aerospace ones but can easily be related to space industry through power systems (batteries) or information technology (intrusion detection). For example, (Saha, Goebel, \& Christophersen, 2009) shows that RVM technique (Bayesian treatment of SVM) outperformed the classical EKF in battery's RUL estimation.

A more detailed comparison for ML tasks is shown in Table 4. Two more criteria are needed to complete that comparison:

- Dealing with the studied problem's non-linearities.

- Accuracy: minimization of dynamical errors.

At this level, it is worthy to say that for some Micro and Nano-satellite configurations particularly the last ones, and with size, computation, and energetic limitations, some FDD stages have to be carried out at ground control centers. We can cite two strategies for that purpose:

- For many micro-satellite configurations, the attitude determination is based on gyro-stellar estimation (see for example (Winkler, Wiedermann, \& Gockel, 2008) and (Torres, Fallet, Peus, Pittet, \& Prieur, 2006)). The gyrostellar estimation suffers when gyroscopes become faulty simultaneously with star-tracker blinding, this situation maximize the attitude pointing error which leads to safe mode switching and mission interruption. The FDI task that follows is executed by ground crew to identify which gyroscope is faulty and to start gyro-less configuration design (e.g. Kalman filter gains tuning).

- For interplanetary missions, the communication delay is enormous (e.g. the delay for New Horizons was about 4.5 hours when close to Pluto), ground FDD tasks had no immediate impact on the mission safety and for that reason, onboard FDIR schemes are very complex. In contrast, and as stated by (Zolghadri, Henry, Cieslak, Efimov, \& Goupil, 2014), for non-critical missions due to their availability rates (Micro and Nano-satellites are often operating at Low Earth Orbits), ground intervention is carried out after safe-mode switching and with explicit analysis of telemetry data to isolate fault source.

After reviewing FDD methods for both model-based and data-driven methods, and to complete the overall control loop to guarantee FTC capabilities, a deep study for the latter has to be carried out.

\section{SPACECraft AtTitude FAult TOLERANT CONTROL}

In the spacecraft attitude control system, and to fulfill the pointing accuracy requirements for missions such as earth observation, the closed-loop control configuration is used in system design. Nevertheless, the performance of such systems decreases when faults affect actuators, sensors, or both. To overcome that limitation, the classical PID or even robust control configurations such as $\mathbb{Z}_{\infty}$ or sliding mode control (SMC) have to be improved to allow fault-tolerant capabilities.

A good introductory FTC study is given earlier in this paper. Thought, a more detailed concept is needed to extend the reader view around FTC in particular for the aerospace field. For that purpose, the detailed architecture of active FTC is drawn in Figure 8 (Alwi, Edwards, \& Tan, 2011).

In the above schematic, the role of the reconfiguration mechanism consists of adapting parameters for both feedback and feed-forward controllers when actuators and sensors faults are detected, isolated, and identified by the FDD module.

As shown in Figure 9, (Alwi, Edwards, \& Tan, 2011) gives a classification of FTC schemes based on their active or passive aspect, to which we add the artificial intelligence-based approaches as an active FTC. Those schemes can be applied to general control engineering including aerospace control systems. Even that sliding mode methodologies are restricted to some types of nonlinear systems, their application in ACS has gained more interest in the literature (Gao Z. , Zhou, Qian, \& Lin, 2018), (Gao Z. , Zhou, Jiang, Qian, \& Lin, 2018), (Hu Q. , 2010), (Lu, Xia, Fu, \& Yu, 2015). On the other hand, AI or data-driven schemes represent a very powerful tool to address FTC (FDD included) problems because they rely mainly on historical data acquired from 
sensors and actuators subsystems. Furthermore, the early detection of some types of faults such as sensor drift can be challenging without inspection about historical sensor data, which emphasizes the application of data-driven methods to meet this kind of challenge (Toubakh, Mouchaweh, Benmiloud, Defoort, \& Djemai, 2020).

Table 3. Learning concept comparison.

\begin{tabular}{|l|c|c|c|c|}
\hline Learning & Labelling & Human supervision & Computing res. & Non-stationarity \\
\hline Supervised & $*$ & $*$ & $* * *$ & $* *$ \\
\hline Semi-supervised & $* *$ & $* *$ & $* *$ & $* *$ \\
\hline Unsupervised & $* * *$ & $* * *$ & $* *$ & $* * *$ \\
\hline Reinforcement & $* * *$ & $* * *$ & $* *$ & $* * *$ \\
\hline Active & $* *$ & $*$ & $* *$ & $* * *$ \\
\hline
\end{tabular}

Table 4. Machine Learning tasks comparison.

\begin{tabular}{|c|c|c|c|c|c|}
\hline \multirow{2}{*}{ Task } & \multirow{2}{*}{ Technique } & \multicolumn{4}{|c|}{ Criteria } \\
\hline & & Non-linearities & Adaptability & Computing res. & Accuracy \\
\hline \multirow{4}{*}{ 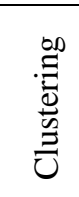 } & k-means & $*$ & $* *$ & $* *$ & $* *$ \\
\hline & A. propagation & $* *$ & *** & $*$ & $* *$ \\
\hline & Hierarchical & $*$ & $*$ & $* *$ & $* * *$ \\
\hline & DBSCAN & $* *$ & $* *$ & $* *$ & $* * *$ \\
\hline \multirow{5}{*}{ 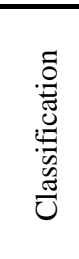 } & SVM & $* *$ & $* *$ & $* *$ & $* * *$ \\
\hline & Logistic. reg & $*$ & $* *$ & $* * *$ & $*$ \\
\hline & N. Bayes & $* *$ & $* *$ & $* * *$ & $* *$ \\
\hline & Class. Trees & $* *$ & *** & $* *$ & $* *$ \\
\hline & $\mathrm{NN}$ & $* * *$ & $* * *$ & $*$ & $* * *$ \\
\hline \multirow{4}{*}{ 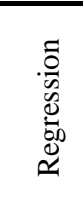 } & Linear. reg & $*$ & $*$ & $* * *$ & $*$ \\
\hline & Reg. Trees & $* *$ & $* *$ & $* *$ & $* *$ \\
\hline & $\mathrm{NN}$ & $* * *$ & $* * *$ & * & $* * *$ \\
\hline & Nearest. $\mathrm{N}$ & $* *$ & $* *$ & $* * *$ & * \\
\hline
\end{tabular}

In this section, several FTC schemes as shown in the academic literature during the last two years will be investigated without discussing the fault detection and isolation aspects as they were extensively studied in the previous sections. In (Bai \& Gao, 2019), the problem of attitude stabilization for rigid spacecraft subject to actuator and sensor faults was addressed. An integral sliding mode controller (ISMC) is proposed to robustly eliminate both the external disturbances and the effects of actuators and sensors' faults. An active fault-tolerant controller (AFTC) was proposed in (Castaldi, Mimmo, \& Simani, 2019). Aerodynamic torques, solar pressure, and magnetic disturbances were considered. The proposed AFTC can compensate for the actuator and sensor fault effect on the closed-loop system using the adaptive control methodology. In (Hu, Liu, Wang, Cheng, \& Luo, 2020), a control switching strategy based on adaptive gain fault observer output is investigated. The adopted ISMC-dual-layer gain adaptation controller improves the fault-related transient performance when compared to other gain adaptation methods. In (Liang, Wang, Hu, \& Dong, 2019), the problem of active FTC for satellite ACS with simultaneous actuator and sensors' faults was addressed. The $\#_{\infty}$ fault-tolerant controller designed in this study has proved its ability to precisely stabilize the attitude angle and angular velocity in the presence of faults. In (Sun L. , 2018), a nonlinear state-feedback controller was derived. The proposed fault-tolerant scheme aims at actuators' loss of effectiveness. A continuously bounded nonlinear function is selected as the controller feature that guarantees the adaptive FTC characteristics. To achieve fault-tolerant capability with vibration suppression for flexible spacecraft, (Zhang, Zong, Tian, \& Liu, 2019) 
proposes a novel scheme based on the continuous multivariable integral sliding mode. The so-called CMISM controller can effectively reject faults during attitude stabilization as well as decrease chattering.

Another critical issue to consider when looking for effective FTC schemes resides in optimization. That is, when faults affect spacecraft ACS actuators or sensors, the attitude control signals have to be optimized against several factors such as:

- $\quad$ The remaining fuel (e.g. hydrazine) in the case of faulty angular rate sensors since they are the main sensors used during orbit maneuvers.

- Optimization is essential to ensure that spacecraft's actuators (e.g. RWs) work without saturation.

- $\quad$ Electric budget must be considered when extra load is acquired by electrical actuators (e.g. magneto-torquers, Control Moment Gyroscopes (CMG)).

Moreover, spacecraft's reentry trajectory must be optimized for some particular missions like Mars/Moon landers or hypersonic vehicles (Chai, et al., 2019).

It is shown in the following, some academic works that address the optimization problem in the spacecraft attitude FTC design. In (Xia, Zhang, Lu, \& Zhou, 2018), the optimized attitude tracking control problem for rigid spacecraft was investigated. The attitude tracking was achieved using sliding mode control (SMC) law. Then, to avoid actuator saturations, a novel method based on dynamic equation optimization is applied. The proposed scheme shows good capabilities allowing faster response and avoiding control input saturations. In addition to variable structure control (VSC), ML and AI-based approaches show promising optimization capabilities for attitude FTC. A deep reinforcement learning approach to improve adaptation characteristics of the spacecraft ACS was introduced in $(\mathrm{Xu}$, $\mathrm{Wu}, \&$ Zhao, 2018). The proposed framework uses a heuristic search to optimize policy. The main drawback of modelbased deep reinforcement learning with heuristic (MDRLH) search algorithm is its tremendous consumption of computing resources which excludes its near implementation on satellites' software configuration. Another AI-based scheme for satellite attitude FTC purposes was reported in (Ning, Xiaodong, Yuanqing, Jie, \& Qiping, 2019). The actuator anti-saturation capability was considered. The proposed approach relies on radial basis function neural networks (RBFNNs). Furthermore, the use of scalars as controllers' adaptive parameters can reduce the computational burden.

Despite the amount of conducted FDD/FTC researches by the academic community (especially for data-driven approaches), their applications in aerospace missions remain limited due to the skeptical view of aerospace industry leaders towards those new techniques (Zolghadri, Henry, Cieslak, Efimov, \& Goupil, 2014).

Indeed, to implement a new FTC approach on an already proven spacecraft configuration, a heavy validation/verification procedure has to be conducted. Such a procedure will, without a doubt, be too expensive in terms of money and time. As a concluding remark, we can highlight that model-based FDD/FTC schemes for space applications represent a mature research field with many contributions that already implemented on real spacecraft (e.g. KF-based gyro-stellar hybridization). Nevertheless, they suffer from several limitations such as model availability/fidelity and system's non-stationary behavior during mission-critical phases (e.g. celestial body landing). To overcome the previous drawbacks, novel techniques have to be investigated like data-driven approaches or Artificial Intelligence (AI).

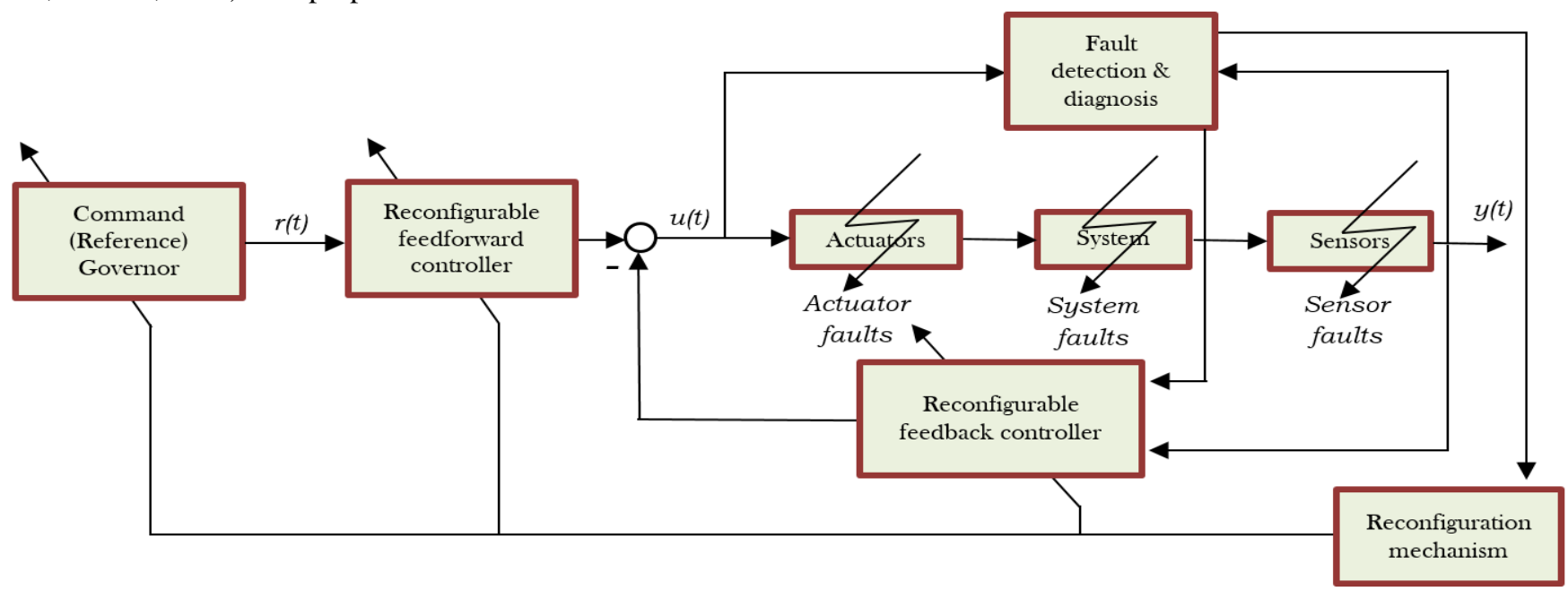

Figure 8. Detailed schematic of active FTC (Alwi, Edwards, \& Tan, 2011). 


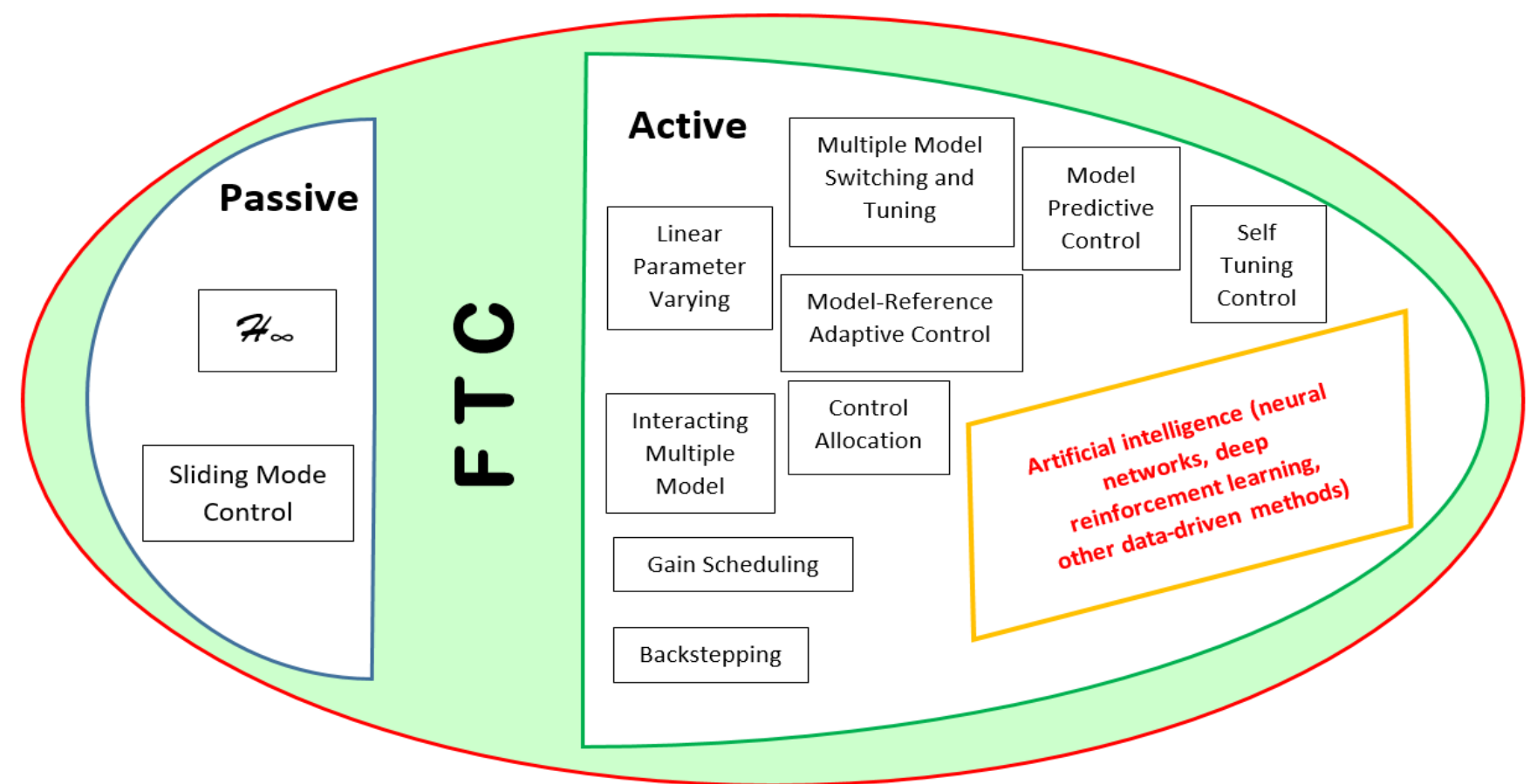

Figure 9. Passive and active FTC classification (adapted from (Alwi, Edwards, \& Tan, 2011)).

Although artificial neural networks (ANN) represent a powerful tool for model-free and non-stationary configurations, they are very greedy to memory resources and CPU load. It exists some "lighter" data-driven techniques such as random forest, linear/logistic regression, SVM, and k-means clustering that can be used as a good compromise for the previous challenges.

At this stage, we can highlight some drawbacks of datadriven strategies. Those strategies suffer from the lack of real failure data to build the classification-based models. Furthermore, data-driven approaches need representative data about each fault behavior. Indeed, many space missions' historical data are not accessible to the academic community, which impacts the quantitative aspect of training data, also from a qualitative perspective during the spacecraft lifetime; a complete database of failure scenarios could not be available due to the reliability of the components, security and safety conditions or because of the very high costs to obtain them. In addition, since the data-driven approaches are black-box, they cannot provide an interpretation to their output allowing to certify it or explain the causes that generate it, especially with neural network schemes, and due to their "black box" structure, the internal behavior cannot be easily understood (Patton, Chen, \& Chen, 2000). This is a major problem for critical systems such as satellite attitude control. An alternative to avoid these two major drawbacks is the use of model-based approaches. They provide a physical interpretation and a formal verification of their output since they are based on the use of physical law principals about the system dynamics and behavior. Indeed, model-based methods due to their widespread use in aerospace engineering (e.g. estimation problems using Kalman filtering), and in the literature (e.g. sliding mode observer/controller), remain the most used contemporary tool to design onboard FTC/FDD architectures. For example, using Kalman filters, which made famous after their application in NASA's Apollo program, optimal estimation solution is achieved with simple implementation in contrast to complicated AI schemes (e.g. $\mathrm{NN}$ ). Also, the recursive form of KF guarantees the simple formulation of online processing. Another advantage of model-based methods referring to the previous paragraph is their simple transportability and reapplication throughout many spacecraft of the same family (e.g. Myriade), or even the adaptation to a different configuration without complicated study.

An alternative in order to overcome the drawbacks of datadriven and physical model based approaches is to combine them through a hybrid model that gathers their advantages (Yin, Xiao, Ding, \& Zhou, 2016), (Lughofer \& M SayedMouchaweh, 2019), (Djeziri, Benmoussa, Mouchaweh, \& Lughofer, 2020). Indeed, a physical model can be generated using the available physical knowledge about the system's dynamics and configuration modes. Then, this model is enriched and reinforced over time by using incoming data about the system operation conditions and environments.

At the end of FTC methods review, it is important to mention that despite the enormous amount of academic papers focusing on ACS FTC, the basic strategy of safe mode transition as outlined in (Wander \& Förstner, 2012), (Yin, 
Xiao, Ding, \& Zhou, 2016), still widely used (ESA and NASA's several missions) when critical faults are detected by on-board FDIR, and the mission recovery is guaranteed afterwards by the ground crew. The dependability on safe mode transition shows that none of the FTC reviewed works can fully cope with all spacecraft critical situations. According to (Wander \& Förstner, 2012), cognitive methods represent a promising tool to meet that challenge.

\section{CONCLUSION}

In this paper, an overview of recent FDD and FTC methodologies proposed for satellite ACS was elaborated. The need for efficient satellite attitude FTC system escalates with the sophistication of the space systems recently developed. That is, the precision, stability, and real-time reaction in faulty cases are required. Indeed, it was shown throughout this paper that data-driven approaches have proven their superiority over model-based ones, particularly when addressing FDD/FTC problems in non-stationary environments. Another key feature resides in artificial intelligence capability to deal with system dynamics nonlinearities. During this study, we have seen that some challenges of data-driven based FTC need to be addressed, especially when investigating the problem of huge computational resources and memory space. Fortunately, the aforementioned problem can be solved by applying statistical summarization techniques and dimension reduction techniques. Finally, knowing that an efficient FDD/FTC scheme has to ensure optimized performance in the space environment, we have seen in the last subsection that AI approaches have the best capacity of optimization, in particular, using deep reinforcement learning. As future work, and based on data-driven attitude FTC challenges identified in this paper, we focus on addressing attitude sensor's FDD using a new data-driven or AI approach with high and optimized performance.

\section{REFERENCES}

Ahmad, I., Basheri, M., Iqbal, M., \& Raheem, A. (2018). Performance comparison of support vector machine, random forest, and extreme learning machine for intrusion detection. IEEE Access, 6, 33789-33795. doi:10.1109/access.2018.2841987

Alwi, H., Edwards, C., \& Marcos, A. (2010). FDI for a Mars orbiting satellite based on a sliding mode observer scheme. Conference on Control and Fault Tolerant Systems, (pp. 125-130). Nice, France.

Alwi, H., Edwards, C., \& Tan, C. P. (2011). Fault Detection and Fault-Tolerant Control Using Sliding Modes. Springer. doi:10.1007/978-0-85729-650-4

Bai, L., \& Gao, Z. (2019). Integrated Fault-Tolerant Stabilization Control for Satellite Attitude Systems with Actuator and Sensor Faults. Journal of
Control, Automation and Electrical Systems. doi:10.1007/s40 313-019-00498-3

Baldi, P., Blanke, M., Castaldi, P., Mimmo, N., \& Simani, S. (2018). Fault diagnosis for satellite sensors and actuators using nonlinear geometric approach and adaptive observers. International Journal of Robust and Nonlinear Control. doi:10.1002/rnc.4083

Byeon, S., Mok, S.-H., Woo, H., \& Bang, H. (2019). SensorFault Tolerant Attitude Determination Using TwoStage Estimator. Advances in Space Research, 3632-3645. doi:10.1016/j.asr.2019.02.007

Castaldi, P., Mimmo, N., \& Simani, S. (2019). LEO satellite active FTC with aerodynamic disturbance decoupled fault diagnosis. European Journal of Control. doi:10.1016/j.ejcon.2019.06.005

Chai, R., Tsourdos, A., Savvaris, A., Chai, S., Xia, Y., \& Chen, C. L. (2019). Six-DOF Spacecraft Optimal Trajectory Planning and Real-Time Attitude Control: A Deep Neural Network-Based Approach. IEEE Transactions on Neural Networks and Learning System.

Djebko, K., Puppe, F., \& Kayal, H. (2019). Model-Based Fault Detection and Diagnosis for Spacecraft with an Application for the SONATE Triple Cube NanoSatellite. doi:10.3390/aerospace6100105

Aerospace.

Djeziri, M. A., Benmoussa, S., Mouchaweh, M. S., \& Lughofer, E. (2020). Fault diagnosis and prognosis based on physical knowledge and reliability data: Application to MOS Field-Effect Transistor. Microelectronics Reliability. doi:10.1016/j.microrel.2020.113682

Ducard, G. J. (2009). Fault-tolerant Flight Control and Guidance Systems, Practical Methods for Small Unmanned Aerial Vehicles. Springer.

ESA. (1999). SOHO's Recovery: An Unprecedented Success Story. European Space Agency. Bulletin 97 (Mar.1999).

Fault Detection Method of Luojia1-01 Satellite Attitude Control System Based on Supervised Local Linear Embedding. (2019). IEEE Acces.

Gao, S., Zhang, W., \& He, X. (2018). Observer-Based Multiple Faults Diagnosis Scheme for Satellite Attitude Control System. Asian Journal of Control. doi:10.1002/asjc. 1873

Gao, S., Zhang, Z., Zhang, W., He, X., \& Lu, X. (2019). Fault Diagnosis for Satellite Attitude Control System with Using Extended Kalman Filter. 38th Chinese Control Conference (CCC), (pp. 4789-4794). doi:10.23919/chicc.2019.8865903

Gao, Z., Zhou, Z., Jiang, G., Qian, M., \& Lin, J. (2018). Active Fault Tolerant Control Scheme for Satellite Attitude Systems: Multiple Actuator Faults Case. International Journal of Control, Automation and Systems, 1794-1804. doi:10.1007/s12555-01 
Gao, Z., Zhou, Z., Qian, M. S., \& Lin, J. (2018). Active fault tolerant control scheme for satellite attitude system subject to actuator time-varying faults. IET Control Theory \& Applications. doi:10.1049/ietcta.2017.0969

Gao, Z., Zhou, Z., Qian, M. S., \& Lin, J. (2018). Active fault tolerant control scheme for satellite attitude system subject to actuator time-varying faults. IET Control Theory \& Applications, 405-412. doi:10.1049/ietcta.2017.0969

Gao, Z.-F., Jiang, B., Shi, P., \& Cheng, Y.-H. (2010). Sensor Fault Estimation and Compensation for Microsatellite Attitude Control Systems. International Journal of Control, Automation, and Systems, 228-237. doi:10.1007/s12555-010-0207-7

García-Gutiérrez, J., Martínez-Álvarez, F., Troncoso, A., \& Riquelme, J. (2015). A comparison of machine learning regression techniques for LiDAR-derived estimation of forest variables. Neurocomputing, 167, 24-31. doi:10.1016/j.neucom.2014.09.091

Hasan Abbasi, N., Castaldi, P., HamedDehghan, B., \& Simani, S. (2019). Novel Non-Model-Based Fault Detection and Isolation of Satellite Reaction Wheels Based on a Mixed-Learning Fusion Framework. IFAC PapersOnline, 194-199. doi:10.1016/j.ifacol.2019.11.222

Hassanien, A. E., Darwish, A., \& El-Askary, H. (2020). Machine Learning and Data Mining in Aerospace Technology. Springer. doi:10.1007/978-3-03020212-5

Henry, D. (2008). Fault Diagnosis of Microscope Satellite Thrusters Using Hinf/H_ Filters. JOURNAL OF GUIDANCE, CONTROL, AND DYNAMICS, 699711. doi:10.2514/1.31003

Hu, H., Liu, L., Wang, Y., Cheng, Z., \& Luo, Q. (2020). Active fault-tolerant attitude tracking control with adaptive gain for spacecrafts. Aerospace Science and Technology. doi:10.1016/j.ast.2020.105706

$\mathrm{Hu}$, Q. (2010). Robust adaptive sliding-mode fault-tolerant control with L2-gain performance for flexible spacecraft using redundant reaction wheels. IET Control Theory \& Applications, 1055-1070. doi:10.1049/iet-cta.2009.0140

Ibrahim, S. K., Ahmed, A., Zeidan, E. M., \& Ziedan, I. E. (2019). Machine Learning Techniques for Satellite Fault Diagnosis. Ain Shams Engineering Journal. doi:10.1016/j.asej.2019.08.006

JAXA. (2016). Supplemental Handout on the Operation Plan of the X-ray Astronomy Satellite ASTRO-H (Hitomi). JAXA. 28 April 2016.

Ke-Qiang, X., Meng, X., Jun, W., Bao-Jun, L., Zhuo, C., \& Gan-Hua, L. (2019). A New Method for Satellite Control System Fault Pattern Recognition combining Multi-Classification SVM with Kernel Principal Component Analysis. IEEE 3rd Information Technology, Networking, Electronic and Automation Control Conference (ITNEC), (pp. 794-797). doi:10.1109/itnec.2019.8729412

Lee, K.-H., Lim, S., Cho, D.-H., \& Kim, H.-D. (2020). Development of Fault Detection and Identification Algorithm Using Deep learning for Nanosatellite Attitude Control System. International Journal of Aeronautical and Space Sciences. doi:10.1007/s42405-019-00235-9

Li, G., Li, J., Cao, Y., Xu, M., Xia, K., Wei, J., \& Dong, L. (2019). The flywheel fault detection based on Kernel principal component analysis. IEEE 3rd Information Technology, Networking, Electronic and Automation Control Conference (ITNEC), (pp. 425-432). doi:10.1109/itnec.2019.8729163

Li, L., Liu, C., Zhang, Y., Wang, Z., \& Shen, Y. (2019). Gyroscope fault accommodation based on dedicated Kalman filters. Journal of Aerospace Engineering. doi:10.1177/0954410019861188

Liang, X., Wang, Q., Hu, C., \& Dong, C. (2019). Observerbased $\mathrm{H} \infty$ fault-tolerant attitude control for satellite with actuator and sensor faults. Aerospace Science and Technology. doi:10.1016/j.ast.2019.105424

Liu, Y., Pan, Q., Wang, H., \& He, T. (2019). Fault diagnosis of satellite flywheel bearing based on convolutional neural network. Prognostics and System Health Management Conference. Qingdao. doi:10.1109/phm-qingdao46334.2019.8942957

Lopez-Encarnacion, E., Fonod, R., \& Bergner, P. (2019). Model-based FDI for Agile Spacecraft with Multiple Actuators Working Simultaneously. IFAC PapersOnLine, 436-441. doi:10.1016/j.ifacol.2019.11.282

Lu, K., Xia, Y., Fu, M., \& Yu, C. (2015). Adaptive finitetime attitude stabilization for rigid spacecraft with actuator faults and saturation constraints. International Journal of Robust and Nonlinear Control, 28-46. doi:10.1002/rnc.3289

Lughofer, E., \& M Sayed-Mouchaweh. (2019). Predictive maintenance in dynamic systems: advanced methods, decision support tools and real-world applications. Springer.

Mehra, R., Rago, C., \& Seereeram, S. (1998). Autonomous Failure Detection, Identification and Fault-tolerant Estimation with Aerospace Applications., (pp. 133138). doi:10.1109/aero.1998.687904

Nagesh, I., \& Edwards, C. (2011). A Sliding Mode Observer Based FDI Scheme for a Nonlinear Satellite. IEEE International Conference on Control Applications (CCA). doi:10.1109/cca.2011.6044485

Nemati, F., Safavi Hamami, S., \& Zemouche, A. (2019). A nonlinear observer-based approach to fault detection, isolation and estimation for satellite formation flight application. Automatica, 474-482. doi:10.1016/j.automatica.2019.06.007 
Ning, Z., Xiaodong, C., Yuanqing, X., Jie, H., \& Qiping, W. (2019). Quaternion-Based Fault-Tolerant Control Design for Spacecraft Attitude Stabilization: An Anti-Saturation Method. Chinese Control Conference doi:10.23919/chicc.2019.8866098

$(C C C)$.

Omran, E. A., \& Murtada, W. A. (2017). Efficient anomaly classification for spacecraft reaction wheels. Neural Computing and Applications. doi:10.1007/s00521017-3226-y

Patton, R. J., Chen, J., \& Chen, J. (2000). A study on neurofuzzy systems for fault diagnosis. International Journal of Systems Science, 31(11), 1441-1448. doi:10.1080/00207720050197811

Qu, Z., Xu, K., Chen, Z., He, X., Xie, Y., Liu, M., . . Han, S. (2019). Fault Detection Method of Luojia1-01 Satellite Attitude Control System Based on Supervised Local Linear Embedding. IEEE Acces. doi:10.1109/access.2019.2932392

Rouabah, B., Toubakh, H., \& Mouchaweh, M. (2020). Fault tolerant control of multicellular converter used in shunt active power filter. Electric Power Systems Research, 188. doi:10.1016/j.epsr.2020.106533

Saha, B., Goebel, K., \& Christophersen, J. (2009). Comparison of prognostic algorithms for estimating remaining useful life of batteries. Transactions of the Institute of Measurement and Control, 31(3/4), 293-308. doi:10.1177/0142331208092030

Samy, I., \& Gu, D.-W. (2012). Fault Detection and Flight Data Measurement, Demonstrated on Unmanned Air Vehicles Using Neural Networks. Springer.

Sun, B., Wang, J., He, Z., Zhou, H., \& Gu, F. (2019). Fault Identification for a Closed-Loop Control System Based on an Improved Deep Neural Network. Sensors. doi:10.3390/s19092131

Sun, L. (2018). Constrained Adaptive Fault-Tolerant Attitude Tracking Control of Rigid Spacecraft. Advances in Space Research, 2229-2238. doi:10.1016/j.asr.2018.12.021

Torres, A., Fallet, C., Peus, A., Pittet, C., \& Prieur, P. (2006). MYRIADE MICROSATELLITES : CURRENT DEVELOPMENTS AROUND A PROVEN AOCS CONCEPT. 4S Symposium, Small Satellites, Systems and Services. Sardinia, Italy.

Toubakh, H. (2015). PhD. Thesis: Automated on-line early fault diagnosis of wind turbines based on hybrid dynamic classifier. Retrieved from http://www.theses.fr/2015LIL10100/document

Toubakh, H., Mouchaweh, M., Benmiloud, M., Defoort, M., \& Djemai, M. (2020). Self adaptive learning scheme for early diagnosis of simple and multiple switch faults in multicellular power converters. ISA Transactions. doi:10.1016/j.isatra.2020.03.025

Wander, A., \& Förstner, R. (2012). Innovative Fault Detection, Isolation and Recovery Strategies onboard Spacecraft: state of the art and Research
Challenges. Deutscher Luft- und Raumfahrtkongress.

Winkler, S., Wiedermann, G., \& Gockel, W. (2008). GYROSTELLAR ATTITUDE ESTIMATION CONSIDERING MEASUREMENT NOISE CORRELATION AND TIME-VARIANT RELATIVE SENSOR MISALIGNMENT. International Astronautical Congress. Glasgow, Scotland.

Wu, D., Wang, H., Liu, H., He, T., \& Xie, T. (2019). Health Monitoring on the Spacecraft Bearings in HighSpeed Rotating Systems by Using the Clustering Fusion of Normal Acoustic Parameters. Applied Sciences. doi:10.3390/app9163246

Wu, S.-F., Engelen, C. J., Babuška, R., Chu, Q.-P., \& Mulder, J. A. (2003). Fuzzy logic based full-envelope autonomous flight control for an atmospheric reentry spacecraft. Control Engineering Practice, 1125. doi:10.1016/s0967-661(02)00075-8

Wu, S.-F., Engelen, C., Chu, Q.-P., Babuška, R., Mulder, J., \& Ortega, G. (2001). Fuzzy logic based attitude control of the spacecraft X-38 along a nominal reentry trajectory. Control Engineering Practice, 699 707. doi:10.1016/s0967-0661(01)00036-3

Xia, Y., Zhang, J., Lu, K., \& Zhou, N. (2018). Finite-Time Fault Tolerant Attitude Tracking Control for Rigid Spacecraft with Actuator Saturation. Advances in Industrial Control, 93-116. doi:10.1007/978-98113-1373-8_6

Xu, K., Wu, F., \& Zhao, J. (2018). Model-based deep reinforcement learning with heuristic search for satellite attitude control. Industrial Robot: An International Journal. doi:10.1108/IR-05-20180086

Yin, S., Xiao, B., Ding, S. X., \& Zhou, D. (2016). A Review on Recent Development of Spacecraft Attitude Fault Tolerant Control System. IEEE Transactions on Industrial Electronics, 3311-3320. doi:10.1109/tie.2016.2530789

Yun, Y., Lee, J., Oh, H., \& Choi, J.-H. (2019). Remaining useful life prediction of reaction wheel motor in satellites. JMST Advances. doi:10.1007/s42791019-00020-5

Zhang, X., Zong, Q., Tian, B., \& Liu, W. (2019). Continuous robust fault-tolerant control and vibration suppression for flexible spacecraft without angular velocity. Int J Robust Nonlinear Control, 39153935. doi:10.1002/rnc.4584

Zhang, Y., \& Jiang, J. (2008). Bibliographical review on reconfigurable fault-tolerant control systems. Annual Reviews in Control, 229-252. doi:10.1016/j.arcontrol.2008.03.008

Zolghadri, A., Henry, D., Cieslak, J., Efimov, D., \& Goupil, P. (2014). Fault Diagnosis and Fault-Tolerant Control and Guidance for Aerospace Vehicles. From Theory to Application. Springer. 